\title{
PRÁCTICAS TUTELADAS EN FARMACIA
}

\section{SUPERVISED PRACTICE OF PHARMACY}

\section{AUTORES}

Xosé García Jiménez: Profesor de farmacología en la Universidad de Alcalá de Henares (España) xose.garcia@arrakis.es

\section{CURRÍCULUM VITAE}

Profesor de farmacología en la Universidad de Alcalá de Henares (España) y miembro del Claustro de dicha Universidad. Autor de artículos científicos sobre química inorgánica y farmacopea, así como ponente y conferenciante en numerosos Congresos nacionales e Internacionales.

\section{RESUMEN}

Tras la información difundida en el número anterior de Vivat Academia, referente al conflicto surgido entre los alumnos y el Decanato de la Facultad de Farmacia de la Universidad de Alcalá, con motivo de la exigencia europea de aumentar las horas dedicadas a las prácticas tuteladas, les presentamos aquí un documento, facilitado por el Sr. Vicedecano de dicha Facultad, D. Taisir Masoud, que contiene algunos aspectos de las negociaciones llevadas a cabo entre los responsables nacionales del problema

\section{PALABRAS CLAVE}

Pácticas tuteladas - Farmacia - Docencia - Investigación 


\section{ABSTRACT}

After the information disclosed in the previous issue of Vivat Academia, regarding the conflict between students and the Dean of the Faculty of Pharmacy, University of Alcala, on the occasion of the European demand to increase the hours devoted to supervised practice, we present here a document provided by Mr. Dean of Faculty said, D. Taisir Masoud, which contains some aspects of the negotiations held between the national leaders of the problem

\section{KEY WORDS}

Pactice supervised - Pharmacy - Teaching - Research

\section{ÍNDICE}

1. Informe sobre aspectos académicos relacionados con las prácticas tuteladas de Farmacia

1.1. Informe y Recomendaciones sobre el periodo de prácticas como elemento de formación de los farmacéuticos (III/F/5289/8/90_ES)

1.2. Número de créditos correspondientes al período de prácticas según el Estado Español

\subsection{Real Decreto $1267 / 1994$}

1.4. Resolución de 18 de Noviembre de 1996 de la Secretaría de Estado de Universidades, Investigación y Desarrollo

2. Conclusiones y posibilidades

3. Las soluciones que proponen los Decanos

4. Nuestros comentarios 
Tras la información difundida en el número anterior de Vivat Academia, referente al conflicto surgido entre los alumnos y el Decanato de la Facultad de Farmacia de la Universidad de Alcalá, con motivo de la exigencia europea de aumentar las horas dedicadas a las prácticas tuteladas, les presentamos aquí un documento, facilitado por el Sr. Vicedecano de dicha Facultad, D. Taisir Masoud, que contiene algunos aspectos de las negociaciones llevadas a cabo entre los responsables nacionales del problema.

1. Informe sobre aspectos académicos relacionados con las prácticas tuteladas de Farmacia

\author{
ANTECEDENTES: \\ Directiva 432/85 de la Comunidad Europea
}

Art.2

3) "el diploma, certificado, sancionará un ciclo de formación de al menos cinco años y que comprenda:

- al menos cuatro años con dedicación plena en una universidad...

- al menos seis meses de periodo de prácticas en una oficina de farmacia abierta al público o en un hospital bajo la supervisión del servicio farmacéutico de dicho hospital."

5) el ciclo de formación contemplado en el apartado 3) comprende la enseñanza teórica y práctica al menos de las siguientes materias. Botánica...Legislación y, en su caso, Deontología. 


\section{A. Informe y Recomendaciones sobre el periodo de prácticas como elemento de formación de los farmacéuticos (III/F/5289/8/90_ES)}

4. "La duración mínima de las prácticas y los lugares en los que deben realizarse... y deben incorporarse a las normas nacionales en la forma pertinente.

4.1. La formación práctica (periodo de prácticas) debe adquirirse bajo la supervisión de farmacéuticos experimentados y capacitados a estos efectos.

4.2. La formación debe tener lugar sólo en las farmacias abiertas al público o en departamentos farmacéuticos de hospitales reconocidos por los organismos de los Estados miembros encargados de la supervisión de las prácticas.

4.3. Los organismos competentes para la supervisión de las prácticas deben establecer claramente los objetivos del mismo. Así, debe garantizarse, entre otras, que el farmacéutico en prácticas...

4.6. Por lo que respecta a la capacitación profesional (cualificación): la evaluación de la capacidad durante el periodo de prácticas y al final del mismo debe hacerse en consonancia con las directrices recogidas en el anexo 7.

Anexo 5. Programa sobre el contenido del periodo de prácticas: Bélgica..., Dinamarca..., ..., España: Cada Universidad desarrolla su propio programa en cooperación con la asociación farmacéutica regional, por lo que en el futuro existirá un programa académico general que formará parte del contrato 
general que tendrán que firmar el Consejo de las Universidades y el Consejo General de las oficinas de farmacia abiertas al público.

Anexo 6. Control de calidad y evaluación del periodo de prácticas. Bélgica..., Dinamarca..., ..., España: Designación del director de la formación práctica: Consejo Universidad (?). Certificación de realización: Universidad. Examen final del periodo de prácticas: No hay ninguna disposición al respecto.

Anexo 7. ... Ello requiere: pruebas del rendimiento del candidato - la valoración de dichas pruebas conforme a los requisitos contenidos en los criterios de rendimiento. Los candidatos no reciben calificaciones, sino que se determina únicamente si están capacitados para desarrollarlas actividades al nivel exigido o si necesitan un periodo adicional de formación enseñanza o prácticas...

B. Número de créditos correspondientes al período de prácticas según el Estado Español

"ESTANCIAS"

Créditos teóricos: --

Créditos prácticos: 15

Créditos total: 15

Descriptor: Periodo de formación de seis meses de prácticas tuteladas que se realizará en oficinas de farmacia. (?) Servicios de farmacia hospitalaria y Empresas de fabricación de medicamentos (????), ajustado a lo determinado en esas directrices generales y en el artículo 1.2 de la Directiva 85/432 de la CEE. 
Áreas de conocimiento: -- No se especifican

NÚMERO DE CRÉDITOS CORRESPONDIENTES AL PLAN DE ESTUDIOS (Ver R.D. 1267/94)

Créditos totales (Directrices generales propias)

Primer ciclo....................................................................95

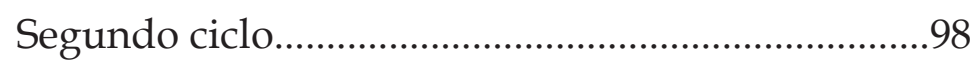

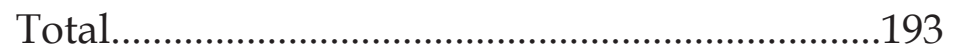

\section{Real Decreto 1267/1994}

Artículo primero

Apartado 7 del Artículo segundo

"7 Crédito... Los excesos de tiempo que puedan implicar las correspondencias a que se refieren los dos párrafos anteriores, no se computarán a efectos del límite máximo de horas a que se refiera el artículo $6^{\circ} 1$. No obstante, las Universidades podrán ampliar el curso escolar que hayan establecido con carácter general, al objeto de que puedan cursarse adecuadamente...

2. Párrafo primero del apartado 3 del artículo tercero

"3. El primer ciclo de las enseñanzas universitarias tendrá una duración de dos a tres años académicos según establezcan, en su caso, las correspondientes directrices generales propias".

3. Párrafo primero del apartado 4 del artículo tercero 
"4 El segundo ciclo... tendrá una duración de dos años académicos. No obstante,... podrán, con carácter excepcional, establecer una duración de hasta tres años..."

Artículo quinto

"El R.D. 1464/1990 ... conducentes a la obtención de (título universitario oficial de Licenciado en Farmacia) mantendrá su vigencia, salvo la directriz segunda del anexo, cuyo párrafo segundo del apartado 1 se suprime y su apartado 2 se modifica, quedando redactado de la forma siguiente:

2 Las Universidades establecerán ... las correspondencias extraordinarias del crédito necesarias para garantizar la formación prevista en la Directiva comunitaria..."

D. Resolución de 18 de Noviembre de 1996 de la Secretaría de Estado de Universidades, Investigación y Desarrollo

... los planes de estudio... licenciado en Farmacia, deben incluir las Estancias en una oficina de farmacia exigidas por la Directiva 85/432/CEE...

\section{Conclusiones y posibilidades}

A. De la Directiva comunitaria no se deduce que la actividad "Estancias" pueda considerarse como una materia troncal del plan de estudios (No se incluye como materia perteneciente al ciclo formativo) comentario 1

B. No parece que esta actividad deba someterse a evaluación, "en el sentido académico clásico" comentario 2

C. No está definido el programa formativo comentario 3 
D. No queda definido el tipo de profesorado que debe "impartir" esta actividad ni si debe estar adscrito a "áreas de conocimiento" comentario 4

E. ¿Cómo deben interpretarse las expresiones "supervisión", "capacitación" y "hospitales reconocidos" referidas a los centros receptores: comentario 5 


\section{Las soluciones que proponen los Decanos}

En la búsqueda de posibles soluciones al problema planteado, podrán establecerse los siguientes supuestos:

A. Sin modificación de Directrices.

La modificación del Decretos de Directrices Generales (RD 1267/94), permite, en principio los siguientes modelos a) $2+(2+1 / 2)+1 / 2$ (900 horas) o b) $(2+1 / 2)+2+1 / 2$. Se requiere una equivalencia 1 crédito P.T. $=60$ horas y una modificación del calendario escolar. Debe señalarse una elevada densidad de la titulación.

Esta opción o supuesto comporta el necesario tratamiento de "Estancias" como una materia más del plan de estudios $y$, en consecuencia, requiere una evaluación académica, la adscripción de profesorado a áreas de conocimiento y Departamentos, la existencia, o no, de requisitos de matrícula, etc.

Por otra parte, debe tenerse en cuenta el problema que plantea el cumplimiento de horarios distintos en los distintos tipos de centros receptores (hospital 8 a 3 / Oficina 9,30 a 13.30 - 4 a 8, etc.

B. Con modificación de Directrices (supresión de Estancias como materia troncal y otras adecuaciones necesarias), pero manteniendo duración total de cinco años.

Si del hecho de que Estancias no figure como materia troncal puede deducirse desaparece el concepto "carga lectiva" para esta actividad, puede pensarse en un modelo $(4+1 / 2)+1 / 2$. En este supuesto se modifican los créditos totales de 
las Directrices propias (193-15=178) (no posible según Directrices Generales por organizar un ciclo con una duración menor a 2 cursos).

C. Con modificación de Directrices (supresión de estancias como materia troncal y otras adecuaciones necesarias con una duración total de 5 años + medio de prácticas tuteladas.

Cinco cursos dedicados a materias troncales (materias del ciclo formativo según Directiva) obligatorias, optativas, etc., más medio año destinado a la realización de prácticas tuteladas. Este modelo permite diseños del tipo $2+3$ ó $3+2$. Disminuye de forma considerable la "troncalidad" según Directrices.

\section{Comentario 6}

\section{Nuestros comentarios}

Nuestro comentario 1: Esto es una forma de coger el rábano por las hojas, ya que si la Directriz de la CEE obliga a esas "estancias", para reconocer la capacitación de un Licenciado en Farmacia que desee trabajar en Europa, una de dos o se plantean dos licenciaturas diferentes, una conducente a la obtención de un título universitario superior sin opción a la apertura de Farmacia y otra estrictamente diseñada para dicho fin, o se diseña un plan de estudios que permita a los licenciados cualquier actividad profesional relacionada con la industria farmacéutica.

Nuestro comentario 2: Evidentemente se nota que vivimos anclados en el pasado. Parece como si nos asustara que una evaluación, que sí está recogida por la CEE, no deba llevar aparejada una calificación de 0 a 10. Debemos cambiar de mentalidad o, desde luego, difícilmente nos adaptaremos a nuestro ingreso en la Comunidad Europea. Cierto es que en nuestro país eso de capacitación no se ha entendido jamás, y si no véanse los problemas existentes con las oposiciones, que nunca se han 
querido sustituir por un procedimiento de habilitación nacional, como existe en otros países.

Nuestro comentario 3: ¿Qué se supone que hace un farmacéutico en una oficina de farmacia o en una farmacia de hospital? Creemos que las cosas las tienen suficientemente claras los colegios de farmacéuticos. ¿O es que están pensando en la utilización de los estudiantes para menesteres propios de un servicio de limpieza exclusivamente? Desde luego nuestra desconfianza no tiene límites. Además las Directrices europeas hablan de control. Otra cosa es que en las directrices españolas aparezcan periodos de formación en industrias farmacéuticas, lo que esta en contradicción con la Directriz de la CEE. ¿Por qué se ha añadido esa posibilidad? Habrá que pensar que ahí se mueven otros intereses. Está claro que los estudiantes podrán realizar prácticas en industrias farmacéuticas, si así se recoge en el plan de estudios, o se oferta como posibilidad de obtener una formación complementaria, pero eso no servirá para la capacitación del farmacéutico como tal. Creemos que el problema aquí reside en que no queremos enfrentarnos con novedades y preferimos utilizar el método del avestruz de la escondida de cabeza. Repetimos, somos recalcitrantes a la hora de introducir innovaciones. Así nos va. Además basta con adaptar los métodos que utilicen otros países que si cumplan con la norma. ¿O no?

Nuestro comentario 4: Esto ya raya en la insensatez. ¿Qué se pretende? A lo peor están pensando que en las oficinas de farmacia además del farmacéutico, que deberá encargarse de la formación en prácticas, deba haber un profesor de la Universidad. Nosotros creemos que las directrices están claras. Lo que ocurre es que habrá que habilitar los medios para que los farmacéuticos acepten los estudiantes en prácticas, por el método que sea. Aquí cada Universidad o Comunidad Autónoma puede decidir lo que le venga en gana. Lo único que se norma por la comunidad europea es que "las prácticas han de estar supervisadas por un farmacéutico experimentado y capacitado a tal efecto". Seamos serios, toda la vida han existido las "venias docendi", 
aplíquese. Otra cosa es si se debe pagar al supervisor por su actividad. Esa es otra historia que dependerá de los convenios que se suscriban. Qué más dan entonces las áreas de conocimiento. A nosotros nos entra la duda de si no se está "mareando la perdiz" como es tan habitual en nuestro país, cuando no se quiere adoptar una solución a un problema. Señoras y señores hay que ser prácticos.

Nuestro comentario 5: huelga cualquiera que queramos hacer. Efectivamente se está queriendo marear la perdiz, no hay duda.

Nuestro comentario 6: Si leemos detenidamente el Decreto, nos daremos cuenta que está previsto que los créditos de prácticas no se computen al mismo nivel horario que los créditos normales. Reiteramos que no sabemos a que viene ese empeño de hablar de profesorado continuamente. Deberían darse una vuelta por los planes de estudios de Universidades de gran prestigio que prevén las estancias en centros ajenos a ellas, sin necesidad de hablar de evaluaciones. Los supervisores emiten un informe que es estudiado por una comisión del Centro que expide el título y se da por valido o no el trabajo realizado por el alumno. Seguimos anclados en el pasado sin querer mirar al futuro, es realmente triste. Lo del horario ya si que nos descoloca del todo, puestos a sacarles peros podemos también hablar de los diferentes colores de las batas usadas en los hospitales y las oficinas de farmacia. Lo importante es que algún Rector que de verdad se preocupe de la docencia, que es lo que le debe preocupar, se decida por adoptar una solución que esté de acuerdo con las directrices europeas. Ya verán como luego terminan copiando los demás, y quizás mejorándolo, como es natural. Las soluciones son múltiples y se nos ocurren de todo tipo. Pero si pretenden poner de acuerdo a todas las Universidades a los Ministerios de Sanidad y Educación, etc, nunca se llegará a salida alguna. Repetimos, seamos prácticos y hagamos uso de la autonomía universitaria que en este caso nos favorece. Claro que los rectores sólo quieren autonomía para satisfacer sus egos personales, que para eso son magníficos. 
A nosotros esto nos recuerda la guerra que sigue existiendo entre los Hospitales y las Facultades de Medicina respecto de las prácticas en los diferentes servicios hospitalarios. Todo ello deriva de un deseo explícito del profesorado universitario por no perder el control férreo que se tiene de los alumnos y sus actividades. Control que, por otra parte, es totalmente ficticio, ya que todo lo basamos en evaluaciones mediante exámenes que nunca dan idea exacta de los conocimientos y la preparación del alumno. ¿Cuándo vamos a querer cambiar de mentalidad en nuestro país? Por otra parte, si analizamos bien el conflicto nos queda por hacer la siguiente pregunta ¿No será que los colegios de farmacéuticos quieren tomar las riendas de la enseñanza en este caso? 